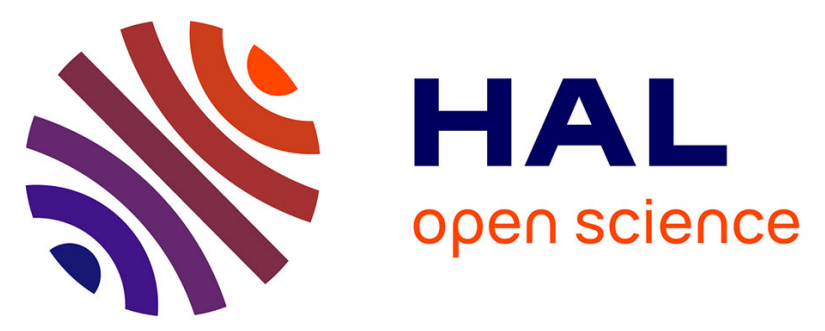

\title{
How different tangible and virtual objects can be while still feeling the same?
}

Xavier de Tinguy, Claudio Pacchierotti, Mathieu Emily, Mathilde Chevalier, Aurélie Guignardat, Morgan Guillaudeux, Chloé Six, Anatole Lécuyer, Maud Marchal

\section{To cite this version:}

Xavier de Tinguy, Claudio Pacchierotti, Mathieu Emily, Mathilde Chevalier, Aurélie Guignardat, et al.. How different tangible and virtual objects can be while still feeling the same?. WHC 2019 IEEE World Haptics Conference, Jul 2019, Tokyo, Japan. pp.580-585, 10.1109/WHC.2019.8816164 . hal-02121839

\section{HAL Id: hal-02121839 \\ https://hal.inria.fr/hal-02121839}

Submitted on 6 May 2019

HAL is a multi-disciplinary open access archive for the deposit and dissemination of scientific research documents, whether they are published or not. The documents may come from teaching and research institutions in France or abroad, or from public or private research centers.
L'archive ouverte pluridisciplinaire $\mathbf{H A L}$, est destinée au dépôt et à la diffusion de documents scientifiques de niveau recherche, publiés ou non, émanant des établissements d'enseignement et de recherche français ou étrangers, des laboratoires publics ou privés. 


\title{
How Different Tangible and Virtual Objects Can Be While Still Feeling the Same?
}

\author{
Xavier de Tinguy ${ }^{1}$, Claudio Pacchierotti ${ }^{2}$, Mathieu Emily ${ }^{4}$, Mathilde Chevalier ${ }^{5}$, Aurélie Guignardat ${ }^{5}$, \\ Morgan Guillaudeux ${ }^{5}$, Chloé $\mathrm{Six}^{5}$, Anatole Lécuyer ${ }^{3}$, and Maud Marchal ${ }^{1}$
}

\begin{abstract}
Tangible objects are used in Virtual Reality to provide human users with distributed haptic sensations when grasping virtual objects. To achieve a compelling illusion, there should be a good correspondence between the haptic features of the tangible object and those of the corresponding virtual one, i.e., what users see in the virtual environment should match as much as possible what they touch in the real world. This paper aims at quantifying how similar tangible and virtual objects need to be, in terms of haptic perception, to still feel the same. As it is often not possible to create tangible replicas of all the virtual objects in the scene, it is important to understand how different tangible and virtual objects can be without the user noticing. This paper reports on the just-noticeable difference (JND) when grasping, with a thumb-index pinch, a tangible object which differ from a seen virtual one on three important haptic features: width, local orientation, and curvature. Results show JND values of $5.75 \%, 43.8 \%$, and $66.66 \%$ of the reference shape for the width, local orientation, and local curvature features, respectively. These results will enable researchers in the field of Virtual Reality to use a reduced number of tangible objects to render multiple virtual ones.
\end{abstract}

\section{INTRODUCTION}

Tangible objects are used in Virtual and Augmented Reality to convey the haptic sensation of touching virtual objects. However, for the illusion to work, the haptic characteristics of the tangible objects should match as much as possible those of the corresponding virtual ones in terms of, e.g., size, local shape, texture, mass. In other words, there should be a good correspondence between what users see in the virtual environment and what they touch in the real world. An easy way to achieve this important visuo-haptic matching is to create tangible replicas of all the virtual objects in the scene. For example, Billinghurst et al. [1] presented an Augmented Reality (AR) tangible book. Users can turn its pages, look at the pictures, and read the text as in a normal book. However, if they use an AR display, they can also see 3-dimensional virtual models popping out of the pages. More recently, Harley et al. [2] presented a system for diegetic tangible objects in Virtual Reality (VR). They developed four tangible objects prototypes, including a cube, a stuffed animal, a treasure chest, and a wooden boat, providing passive and active haptics.

\footnotetext{
*This research has received funding from the EU's H2020 research and innovation programme (grant agreement No 801413, project "H-Reality").

${ }^{1}$ Univ Rennes, INSA, IRISA, Inria, CNRS - Rennes, France \{xavier.de-tinguy, maud.marchal\}@irisa.fr

${ }^{2}$ CNRS, Univ Rennes, Inria, IRISA - Rennes, France claudio.pacchierotti@irisa.fr

${ }^{3}$ Univ Rennes, Inria, CNRS, IRISA - Rennes, France anatole.lecuyerdirisa.fr

${ }^{4}$ Agrocampus Ouest, CNRS, IRMAR - Rennes, France

${ }^{5}$ Agrocampus Ouest - Rennes, France
}

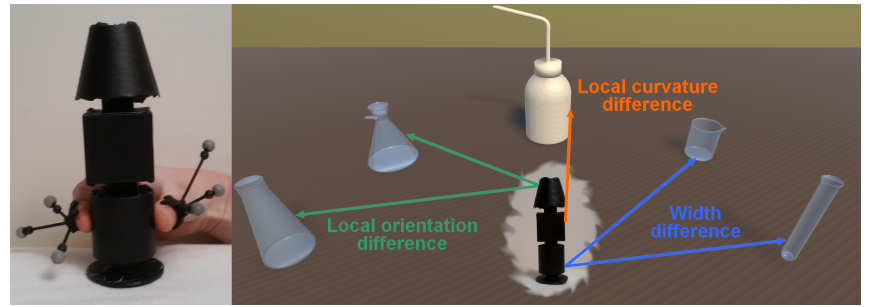

Fig. 1: Objective of our study: understanding how different a tangible object (left) can be from virtual objects (right) without the user noticing the mismatch. We focused our study on three specific criteria: width, local orientation, and curvature.

Altough compelling, creating a tangible replica of all the virtual scene may neither be feasible, e.g., if the virtual objects change dynamically during the experience, nor desirable, e.g., if the virtual environment comprises several objects. For this reason, researchers have recently started to develop solutions enabling the use of few tangible objects to render multiple virtual ones, trying to minimize the haptic mismatch. In this respect, Hettiarachchi and Wigdor [3] introduced a wearable system able to scan the user's surroundings and find a good match between the available virtual and tangible objects. McLelland et al. [4] introduced a reconfigurable device able to change its shape to match the targeted virtual object. It is composed of four rectangular rigid sections with hinged connections. The inFORM [5] system enables experimentation with shape-changing interfaces and dynamic physical affordances. It is made of $30 \times 30$ white polystyrene pins, actuated by push-pull rods that can extend the pins up to $10 \mathrm{~cm}$ from the surface. A projector placed on top of the system can create moving images on the white pins surface and a Kinect depth camera tracks the position of the user's hand. A similar approach has been also presented in [6], where small robots assemble into different shapes. Sait et al. [7], [8] mapped a single tangible object into multiple virtual ones by combining a redirection approach, that uses rotational adjustments to align the user during virtual locomotion, and a resetting approach, that introduces a discrete rotational update when the user virtually approaches a target for interaction. Redirection approaches have also been used in [9], [10].

Although there exists substantial evidence that a mismatch between virtual and physical objects severely affects the user's illusion of presence [11], [12], [13], no one has yet quantitatively studied the extent of this difference in applications of virtual and augmented reality. A first attempt has been carried out by Kwon et al. [14], who explored 
the effects of size and shape differences between tangible and virtual objects for interaction usability in AR. Results showed that manipulation realism increases as the tangible and virtual objects become more similar, but no quantitative measure on the minimum noticeable difference was provided. Similarly, Ban et al. [15] studied the effect of shape difference between tangible and virtual objects in VR. Results show that it is partially possible to alter the shape of the virtual object w.r.t. its tangible counterpart without the user noticing. However, the authors did not provide any quantitative measure of this shape difference. Simeone et al. [11], [16] introduced the concept of Substituional Reality, where every physical object is paired, with some degree of discrepancy, to a contextually-appropriate virtual object. By changing the degree of discrepancy between tangible and virtual objects, the authors highlight several critical mismatches able to break the immersion. For example, results show that tangible objects presenting similar affordances in parts most likely to be interacted with are the best candidates for substitution. Along this line of research, de Tinguy et al. [17] also proposed an algorithm to find similar pinch grasping poses between a set of tangible and virtual objects.

The effect of visuohaptic discrepancy has been also studied from a purely perceptual point of view. For example, Hershberger and Misceo [12] found that, between vision and haptic information, neither modality inherently dominates the perceived size of an object. Discordant haptic information biases visual size estimates by as much as discordant visual information biases haptic size estimates. Since neither modality captures the other one completely, a discordant stimulus will be perceived as discordant. Ernst and Banks [18] quantitatively examined visuohaptic integration to determine whether human performance follows a maximum-likelihood estimate. This rule states that the optimal means of estimation is to sum the sensor estimates weighted by their normalized reciprocal variances. Subjects looked at and/or felt a raised ridge and were asked to judge its height. Results showed that height judgements were indeed very similar to those predicted with a maximum-likelihood estimation. Lacey and Sathian [13] reviewed how the similarities in humans visual and haptic unisensory object processing contribute to an integrated multisensory visuohaptic processing of object in terms of categorization, recognition, and representation. For example, a change in size produces a cost in visual recognition for both unfamiliar and familiar objects. However, neither grasping an object tighter nor enlarging the spread of the fingers leads humans to perceive a change in size [19].

This paper directly addresses the question posed in the title. Our objective is to understand how different a virtual object can be from its tangible counterpart without the user noticing (see Fig. 1). This question is important for all those working in the field of immersive environment, and its answer can open interesting avenues for the use of few tangible objects in the rendering of multiple virtual ones. Of course, the visuohaptic perception of objects encompasses several different dimensions, including the object's size, shape, mass, texture, and temperature. In this work, we started by addressing three representative haptic features - width, local orientation, and curvature, - which are particularly relevant for grasping. We carried out three human subjects experiments, one for each criterion, that we will describe in the next section. A video summarizing our work is available at https://youtu.be/xREuZbh6tLc (7.6Mo, .avi).

\section{Methods}

The goal of our user studies is to measure the JustNoticeable Difference (JND) of the discrepancy between a couple of tangible and virtual objects during 2-finger grasping, in terms of the three above-mentioned haptic dimensions: width, local orientation, and curvature.

\section{A. Experimental setup}

Fig. 2 shows the setup. Participants wear an HTC Vive headset displaying the virtual scene. A Bonita Vicon system tracks the subjects' thumb and index fingertips using markers placed on the dorsal side of their fingers (avoiding the nails). Doing so, the subjects finger pads are always left free to interact with the tangible object (TO).

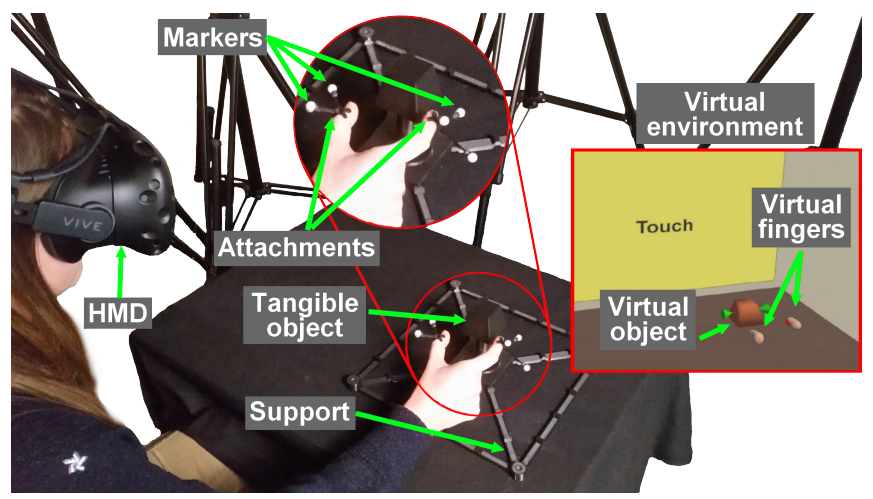

Fig. 2: Setup for the three experiments. Inset shows the virtual environment during the task.

Two virtual fingertips mimic the motion of the subjects fingertips in the virtual environment. This simple representation of the user's hand [20] has been chosen to avoid occluding the virtual object from the user's point of view (see inset of Fig. 2). The virtual scene is composed of an instruction panel and a table, supporting the considered virtual object (VO). The position of the $\mathrm{VO}$ in the virtual scene matches the position of the TO in the real environment. In other words, whenever users grasp the TO, they also grasp the VO. We ensure a good matching between the positions of the TO and VO by securing the TO on a 3D-printed structure, which is placed on a table in front of the user. Moreover, at the beginning of each experiment, we calibrate the system to also ensure a good matching between the subjects fingertips and their virtual avatars.

\section{B. Experimental task and procedure}

Participants are asked to grasp the TO at a designated pinch location, highlighted by two green cursors, while seeing the VO through the headset (see Fig. 2). At every new grasping trial, the system induces a discrepancy between the TO and 
VO by altering the considered criterion, i.e., it creates a mismatch of width, local orientation, or curvature where the subject grasped. We use only one tangible object per experiment, while several different virtual objects are shown to the participants (see Fig. 3). Whenever participants arrive at the highlighted pinch location, the object turns red and a "hold" message is displayed for $2 \mathrm{~s}$ on the virtual panel in front of the user. After that, the object turns green, and the panel asks the user to "release" the object. Right after releasing the object, a question appears on the panel, asking the participants to compare their perception of the considered criteria between the TO and the VO. Finally, the experimenter fakes the changing of the TO, to prevent participants from understanding that only one TO was used throughout the whole experiment. This procedure can be seen in the attached video.

Before the beginning of an experiment, we explain the procedure to the participant and we spend about three minutes adjusting the setup to be comfortable. Then, the participant spend about two minutes practicing interacting with two couples of TO and VO, different than those used during the real trials.
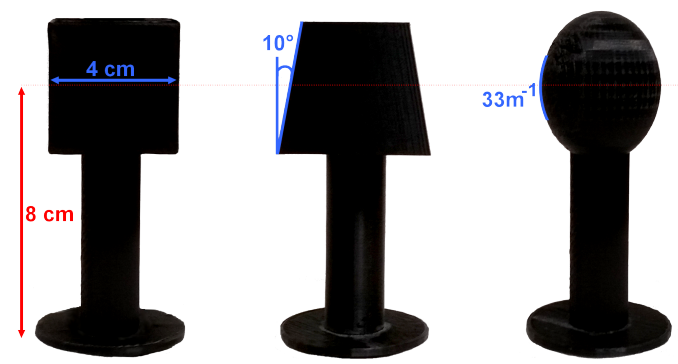

Fig. 3: The tangible objects used for the three user studies: a cube, a trapezoid prism, and an ellipsoid. Participants are asked to grasp at the center of each shape, which is $8 \mathrm{~cm}$ from the table (dotted line).

\section{USER STUDY \# 1: WIDTH}

\section{A. Procedure Description}

The objective of this first user study is to investigate how much the width of a virtual object can differ from the one of a tangible one without the user noticing. We use a cube as the reference shape (left object in Fig. 3), as it is a wellknow shape which is easy to recognize from any point of view. Participants are asked to grasp the virtual object and its tangible counterpart, comparing their width and answering the question: "Is the tangible object larger than the virtual one?"

As mentioned in Sec. II-A, we ensure that whenever the users touch the TO, they also touch the VO. To achieve this result, when the width of the VO and TO differs, we employ a simple virtual warping effect to (slightly) redirect the virtual fingers [10].

\section{B. Experimental Design and Participants}

We consider the cube width as the independent variable, with a reference value for the tangible cube of $w_{\text {ref }}=4.0 \mathrm{~cm}$ (see Fig. 3) and 9 comparison widths for the virtual cubes
$=2.4,3.2,3.6,3.8,4.0,4.2,4.4,4.8,5.6 \mathrm{~cm}$ (see Fig. 4). We compute the comparison widths $w_{\text {ref }}(1+\Delta)$ with $\Delta=$ $\{0 \%, \pm 5 \%, \pm 10 \%, \pm 20 \%, \pm 40 \%\}$. We chose this range of comparison widths so that the covered range contains JND values already registered in the literature [18]. Participants compared each couple of objects 7 times, yielding to $7 \times 9=63$ comparisons per participant. The experiment lasted around 10 minutes.

We enrolled 17 participants ( 9 males, 8 females, $M=$ 21.59, $S D=1.58$ ), all of whom were right-handed.
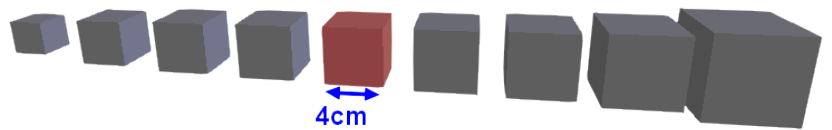

Fig. 4: User study \#1: width. The virtual cubes having variable widths $w_{\text {ref }}(1+\Delta), \Delta=\{0 \%, \pm 5 \%, \pm 10 \%, \pm 20 \%, \pm 40 \%\}$ are compared with the reference cube $\left(w_{\text {ref }}=4.0 \mathrm{~cm}\right)$, shown in red. In the real environment, participants always grasped the tangible cube shown on the left of Fig. 3.

\section{Results}

First, we compute the percentage of answers in which the tangible object felt larger than the virtual object. As expected, as the width of the VO increases, the less often the participants feel the TO larger. Then, using Weber's law, we compute the Weber Fraction as $k=\Delta I / I$, where $\Delta I$ refers to the JND threshold and $I$ is the reference width. The JND threshold can be determined as the value of the stimuli in which the recognition ratio is $75 \%$. To compute this value, we fit the psychometric curve $f(x)=\left(1+e^{(\alpha x+\beta)}\right)$ to the data (with $\alpha=1.12$ and $\beta=-0.4546$ ), as shown in Fig. 5. The $75 \%$ JND is $-0.23 \mathrm{~cm}$, or $5.75 \%$ of the reference width. The corresponding Weber fraction is $k=\Delta I / I=$ 0.0575. Furthermore, the Point of Subjective Equality (PSE) is $0.16 \mathrm{~cm}$.

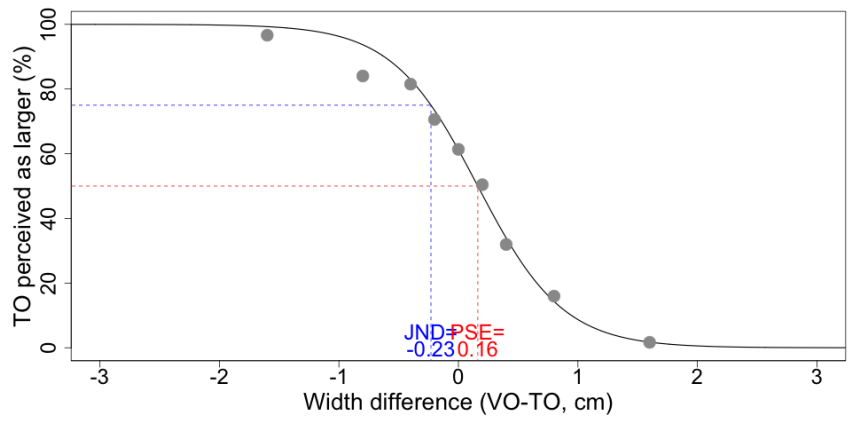

Fig. 5: User study \#1: width. Psychometric curve fitting the average percentage of answers (grey points) in which participants considered the tangible object as larger than the virtual one. The $75 \%$ JND is represented in blue while the PSE is in red.

\section{USER STUDY \#2: LOCAL ORIENTATION}

\section{A. Procedure Description}

The objective of this second user study is to investigate how much the local orientation of a virtual object's face 
can differ from the one of a tangible object without the user noticing. We use a trapezoid prism as the reference shape (center object in Fig. 3). Participants are again asked to grasp the virtual object and its tangible counterpart. Then, they are asked to compare the local orientation at the grasping point, answering to the question: "Are the faces of the tangible object more tilted than those of the virtual one?"

\section{B. Experimental Design and Participants}

We consider the local orientation of the prism faces as the independent variable, with a reference angle for the tangible prism of $a_{r e f}=10^{\circ}$ (see Fig. 3) and 7 comparison angles for the virtual prism $=2^{\circ}, 6^{\circ}, 8^{\circ}, 10^{\circ}, 12^{\circ}, 16^{\circ}, 18^{\circ}$ (see Fig. 6). We compute the comparison angles $a_{r e f}(1+\Delta)$ with $\Delta=$ $\{0 \%, \pm 20 \%, \pm 40 \%, \pm 80 \%\}$. At the pinch location, all virtual prisms have a width of $4 \mathrm{~cm}$. Participants compared each couple of objects 7 times, yielding to $7 \times 7=49$ comparisons for each participant. The experiment lasted around 10 minutes.

We enrolled 17 participants (10 males, 7 females, $M=$ 21.59, $S D=1.58$ ), all of whom were right-handed.
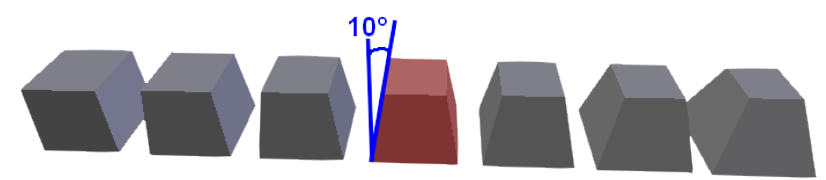

Fig. 6: User study \#2: local orientation. The virtual prisms having variable faces orientations $a_{\text {ref }}(1+\Delta), \Delta=$ $\{0 \%, \pm 20 \%, \pm 40 \%, \pm 80 \%\}$ are compared with the reference prism $\left(a_{r e f}=10^{\circ}\right)$, shown in red. In the real environment, subjects always grasp the tangible prism shown at the middle of Fig. 3.

\section{Results}

First, we compute the percentage of answers in which the tangible object felt more tilted than the virtual object. As before, as the tilting angle of the VO increases, the less often the participants feel the TO as more tilted. Then, we compute the Weber Fraction using the 75\% JND. To compute the latter, we again fit the psychometric curve $f(x)=\left(1+e^{(\alpha x+\beta)}\right)$ to the data (with $\alpha=0.26$ and $\beta=-0.022$ ), as shown in Fig. 7. The $75 \%$ JND is $-4.38^{\circ}$, or $43.8 \%$ of the reference orientation angle. The corresponding Weber fraction is $k=$ 0.438. Furthermore, the PSE is $0.09^{\circ}$.

\section{USER STUdy \#3: LocAl CURVATURE}

\section{A. Procedure Description}

The objective of this third and last user study is to investigate how much the local curvature of a virtual object's faces can differ from the one of a tangible object without the user noticing. We use an ellipsoid as our reference shape (right object in Fig. 3). Participants are again asked to grasp the virtual object and its tangible counterpart. Then, they are asked to compare the curvature at the grasping point, answering to the question: "Are the faces of the tangible object more curved than those of the virtual one?"

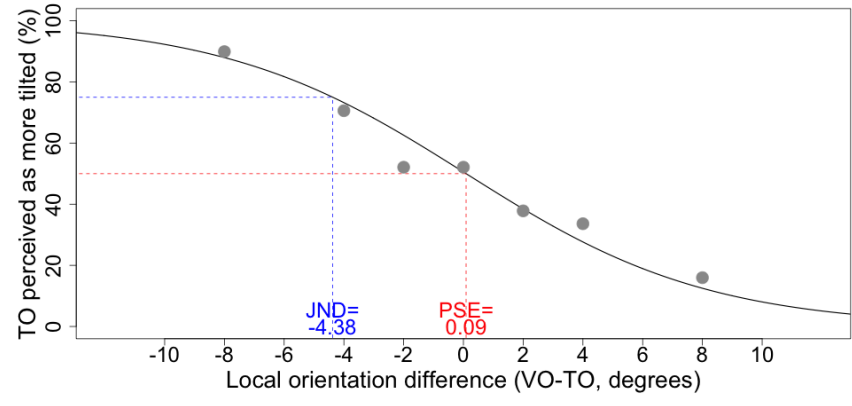

Fig. 7: User study \#2: local orientation. Psychometric curve fitting the average percentage of answers (grey points) in which participants considered the tangible object as more tilted than the virtual one. The $75 \%$ JND is represented in blue while the PSE is in red.

\section{B. Experimental Design and Participants}

The size of the tangible ellipsoid is $4 \times 4 \times 5 \mathrm{~cm}$, with an estimated curvature at the grasping point of $52 \mathrm{~m}^{-1}$ in the horizontal plan and $33 \mathrm{~m}^{-1}$ in the vertical plan. We consider the curvature of the ellipsoid in the vertical plan as the independent variable, with a reference curvature for the tangible ellipsoid of $c_{r e f}=33 \mathrm{~m}^{-1}$ (see Fig. 3) and 9 comparison curvatures for the virtual ellipsoid $=12,19,24,28,33,39,47,72,269 \mathrm{~m}^{-1}$ (see Fig. 8). We compute the comparison curvatures as $c_{r e f}(1+\Delta)$ with $\Delta=$ $\{-63.6 \%,-42.4 \%,-27.3 \%,-15.2 \%, 0 \%,+18.2 \%,+42.4 \%$, $+118.2 \%,+715.2 \%\}$ At the pinch location, all virtual ellipsoids have a width of $4 \mathrm{~cm}$. Participants compared each couple of objects 7 times, yielding to $7 \times 9=63$ comparisons for each participant. The experiment lasted around 10 minutes.

We enrolled 15 participants ( 7 males, 8 females, $M=21.4$, $S D=1.40$ ), all of whom were right-handed.

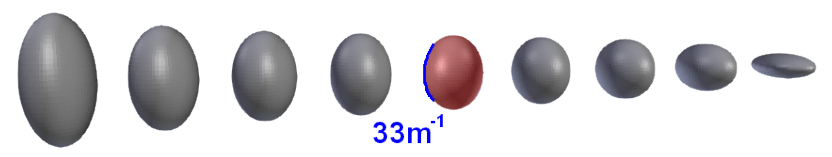

Fig. 8: User study \#3: curvature. The virtual ellipsoids having variable faces curvatures $c_{r e f}(1+\Delta), \Delta=$ $\{-63.6 \%,-42.4 \%,-27.3 \%,-15.2 \%, 0 \%,+18.2 \%,+42.4 \%$, $+118.2 \%,+715.2 \%\}$ are compared with the reference ellipsoid $\left(c_{r e f}=33 \mathrm{~m}^{-1}\right)$, shown in red. In the real environment, participants always grasp the tangible ellipsoid shown in the right of Fig. 3.

\section{Results}

First, we compute the percentage of answers in which the tangible object felt more curved than the virtual object. Then, we compute the Weber Fraction using the 75\% JND. To compute the latter, we fit the psychometric curve $f(x)=$ $\left(1+e^{(\alpha x+\beta)}\right)$ to the data (with $\alpha=0.061$ and $\beta=-1.77$ ), a shown in Fig. 9. The $75 \%$ JND is $22 \mathrm{~m}^{-1}$, or $66.6 \%$ of the reference tilting angle. The corresponding Weber fraction is $k=0.666$. Furthermore, the PSE is $29.04 \mathrm{~m}^{-1}$. 


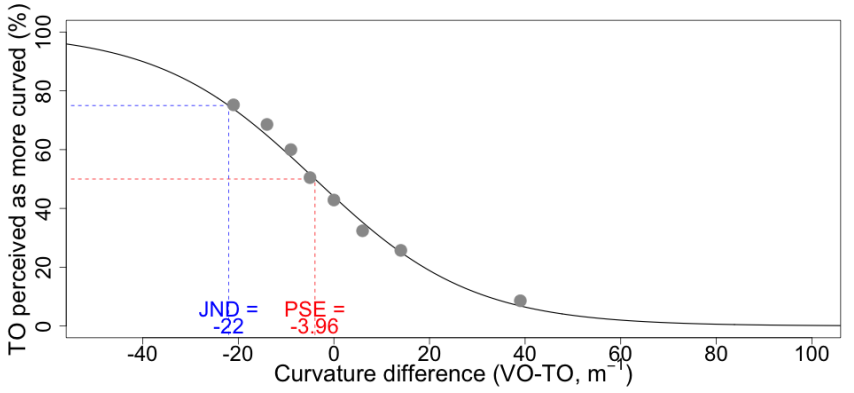

Fig. 9: User study \#3: curvature. Psychometric curve fitting the average percentage of answers (grey points) in which participants considered the tangible object as more curved than the virtual one. The 75\% JND is represented in blue while the PSE is in red. For clarity, the $\Delta=715.2 \%$ value is not represented.

\section{SubJective Questionnaire}

In addition to the three user studies, the participants also fill a subjective questionnaire using a 7-point Likert scale. We asked the following questions: (Q1) "Was it easy to feel the difference of width between the tangible and virtual cubes?"; (Q2) "Was it easy to feel the difference of tilting between the tangible and virtual prisms?"; (Q3) "Was it easy to feel the difference of curvature between the tangible and virtual ellipsoids?"; (Q4) "Did it feel like you were seeing your own fingertips?"; (Q5) "Did you feel tired at the end of the experiment?".

Results show that feeling the difference in width and local orientation was quite easy (Q1: $M=4, S D=1.57$; Q2: $M=3.63, S D=1.21)$. However, it was quite difficult to feel the difference in local curvature $(\mathrm{Q} 3: M=2.84, S D=1.46)$. The corresponding barplots are reported in Fig. 10. The matching between the virtual and the real fingertips appears to be well perceived (Q4: $M=5.11, S D=1.52)$. At the end of the experiment, participants felt a bit tired (Q5: $M=4.05$, $S D=1.68)$.

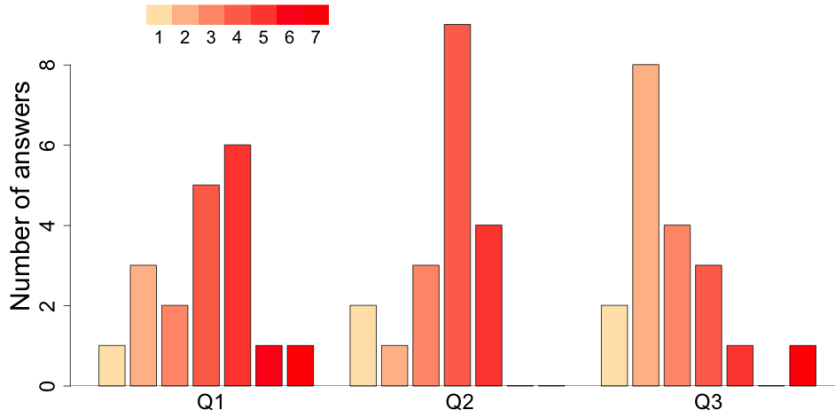

Fig. 10: Barplots (7-point Likert scale) representing the participants answers to the questions on the easiness of perception of the difference between TO and VO for the three criteria: Q1 (width), Q2 (local orientation), Q3 (curvature).

\section{DISCUSSION}

The objective of this paper is to quantify, in a VR scenario, how similar tangible and virtual objects need to be to feel the same. In other words, we wanted to measure how different virtual and tangible objects can be without the user noticing.

Toward this objective, we carried out three human subject experiments. Results showed a 75\% JND of $5.75 \%, 43.80 \%$, and $66.66 \%$ for the perception of width, local orientation, and local curvature during grasping, respectively around references values of $4 \mathrm{~cm}, 10^{\circ}$, and $33 \mathrm{~m}^{-1}$. Those values were selected as central values in the range of realistic values for pinch grasping.

Although no one directly addressed this question for VR, these results are consistent to similar perceptual results in the literature. Ernst and Banks [18] found a 84\% JND of 0.04 times the average ridge height $(55 \mathrm{~mm})$ when providing both visual stimuli. On the other hand, the haptic-only JND was 0.085 times the average height. In our case, the $84 \%$ JND is $10.75 \%$ of the cube size $(40 \mathrm{~mm})$. This difference might be explained by the fact that our participants could see the cube in 3D. Moreover, in this paper we are studying a discrepancy-related JND, which poses a different questions w.r.t. the one considered in [18]. Subjects in Ban et al. [15] were asked to explore a tangible vertical surface with their index finger while seeing a slanted virtual surface on the screen $\left(70^{\circ}\right)$. The ratings of a questionnaire suggest that the two objects (tangible and virtual) felt almost the same. Simeone et al. [11], [16] found that affordance and function are the most important tracts when rendering virtual objects through tangible props. For this reason, they suggest to focus on maximizing the shape similarity around areas the user is likely to interact with (e.g., a handle). Unfortunately, they did not report any quantitative data on how to do this. Finally, subjects in Kwon et al. [14] took 65\% more time to grasp a tangible object when its virtual representation significantly differed. In our experiments, we used the tangible object as reference. It would also be interesting to use the virtual object as reference and change the properties of tangible ones, to observe whether there are any biases. However, as we simulate the change of the tangible object for each repetition of the experiment, we believe that the bias was minimum. Our results open new interesting avenues for the use of tangible objects different from their virtual counterpart. The psychometric curves reported in Secs. III, IV, and V enable researchers to understand when it is acceptable for a tangible object to approximate the haptic features of virtual ones. Doing so, one tangible object can be used to render multiple virtual ones without the user noticing.

From these results, we can also improve our previous work [17], where we proposed an algorithm to find, rate, and extract the best pairs of pinching poses between tangible and virtual objects. Along the same line of thought, we could analyze the haptic features of a virtual environment and then, knowing that it is possible to have some discrepancy, automatically generate one or more universal tangible objects, able to provide the best possible sensations in the rendering of the virtual scene. Alternatively, one could also change the virtual scene to improve the expected visuohaptic matching and, therefore, the illusion of presence (e.g., remove certain types of surfaces which are difficult to render with the 
available tangible objects). It may also enables to predict the illusion of presence and take action if deemed insufficient (e.g., by increasing the number of tangible objects in use).

Although these results are interesting, they are just the beginning of a long line of research. The haptic perception of an object does not only comprise size, local orientation, and curvature. It is therefore important to extend this study to other important haptic features, such as texture, mass, and temperature. More reference values would also be needed to better determine the Weber's fraction, and it would be interesting to do so for each feature as well as for different grasping poses. We also did not take into account that humans have fingertips of different size and elasticity, which can significantly affect how they perceive a surface. Another limitation is that we only considered 2-fingers grasping. We may find different results for other types of interaction. This work also does not directly address any possible confusion due to inherent tracking issue such as occlusions or calibration residual offsets. This issue may be solved by considering different tracking techniques [21] or additional tracking error compensation methods as well as more complex haptic proxy approaches to compensate such case. We will address these points in future work. Finally, we also found that some participants had serious difficulties in recognizing even very large discrepancies. We plan to study this phenomenon in depth, to ensure a minimum guaranteed level of performance for all subjects, even the less perceptive ones.

\section{CONCLUSIONS}

Tangible objects are often used in VR and AR to provide distributed haptic sensations of touching virtual objects. However, it is often not possible to create tangible replicas of all the virtual objects in the scene and one would want to re-use some of those as much as possible, nor is it always possible to create an exact replicate. Thus, it is to be expected that there would be some discrepancy between the tangible objects and the virtual ones. This paper wants to directly address this issue, by measuring how different a virtual object can be from its tangible counterpart without the user noticing. Therefore, we tried to quantify this noticeable perceptual difference between virtual objects and their tangible counterpart during a 2 fingers pinching, by considering the perception of width, local orientation, and local curvature. Although some papers have addressed this problem from a qualitative point of view, to the best of our knowledge, this paper represents the first effort to quantify this noticeable perceptual difference between virtual and tangible objects in virtual reality.

Our experiments show that, while pinching, it was possible to induce discrepancy between what the user feel of the tangible object and what he sees from the virtual one by $5.75 \%, 43.8 \%$, and $66.66 \%$ respectively for the width, local orientation and local curvature. Those results suggest that it is indeed possible, to a certain extent, to match different virtual and tangible objects without the user noticing.

Next steps would be to induce discrepancy on other parameters, such as inertia, texture, roughness, etc. as well as extending the results to other kind of grasping. These efforts would definitely open novel opportunities for using a reduced number of tangible objects to render multiple virtual ones in VR.

\section{REFERENCES}

[1] M. Billinghurst, H. Kato, and I. Poupyrev, "The magicbook-moving seamlessly between reality and virtuality," IEEE Computer Graphics and applications, vol. 21, no. 3, pp. 6-8, 2001.

[2] D. Harley, A. P. Tarun, D. Germinario, and A. Mazalek, "Tangible vr: Diegetic tangible objects for virtual reality narratives," in Proc. of Conference on Designing Interactive Systems, 2017, pp. 1253-1263.

[3] A. Hettiarachchi and D. Wigdor, "Annexing reality: Enabling opportunistic use of everyday objects as tangible proxies in augmented reality," in Proc. of the ACM Conference on Human Factors in Computing Systems, 2016, pp. 1957-1967.

[4] J. C. McClelland, R. J. Teather, and A. Girouard, "Haptobend: Shapechanging passive haptic feedback in virtual reality," in Proc. of 5th Symposium on Spatial User Interaction, 2017, pp. 82-90.

[5] S. Follmer, D. Leithinger, A. Olwal, A. Hogge, and H. Ishii, "inform: dynamic physical affordances and constraints through shape and object actuation." in Proc. ACM UIST, vol. 13, 2013, pp. 417-426.

[6] Y. Zhao, L. H. Kim, Y. Wang, M. Le Goc, and S. Follmer, "Robotic assembly of haptic proxy objects for tangible interaction and virtual reality," in Proc. of the ACM International Conference on Interactive Surfaces and Spaces, 2017, pp. 82-91.

[7] M. S. M. Y. Sait, S. P. Sargunam, D. T. Han, and E. D. Ragan, "Physical hand interaction for controlling multiple virtual objects in virtual reality," in Proc. of the 3rd International Workshop on Interactive and Spatial Computing, 2018, pp. 64-74.

[8] D. T. Han, M. Suhail, and E. D. Ragan, "Evaluating remapped physical reach for hand interactions with passive haptics in virtual reality," IEEE Transactions on Visualization \& Computer Graphics, no. 1, pp. 1-1, 2018.

[9] M. Azmandian, M. Hancock, H. Benko, E. Ofek, and A. D. Wilson, "Haptic retargeting: Dynamic repurposing of passive haptics for enhanced virtual reality experiences," in Proc. of ACM Conference on Human Factors in Computing Systems (CHI), 2016, pp. 1968-1979.

[10] L. Kohli, M. C. Whitton, and F. P. Brooks, "Redirected touching: Training and adaptation in warped virtual spaces," in Proc of IEEE Symposium on 3D User Interfaces, 2013, pp. 79-86.

[11] A. L. Simeone, E. Velloso, and H.-W. Gellersen, "Substitutional reality: Using the physical environment to design virtual reality experiences," in Proc. of ACM Conference on Human Factors in Computing Systems, 2015, pp. 3307-3316.

[12] W. A. Hershberger and G. F. Misceo, "Touch dominates haptic estimates of discordant visual-haptic size," Perception \& Psychophysics, vol. 58, no. 7, pp. 1124-1132, 1996.

[13] S. Lacey and K. Sathian, "Visuo-haptic multisensory object recognition, categorization, and representation," Frontiers in Psychology, vol. 5, p. 730, 2014.

[14] E. Kwon, G. J. Kim, and S. Lee, "Effects of sizes and shapes of props in tangible augmented reality," in Proc. of IEEE International Symposium on Mixed and Augmented Reality, 2009, pp. 201-202.

[15] Y. Ban, T. Narumi, T. Tanikawa, and M. Hirose, "Magicpot360: Free viewpoint shape display modifying the perception of shape," in Proc. of IEEE Internation Conference on Virtual Reality, 2015, pp. 321-322.

[16] A. L. Simeone, "Substitutional reality: Towards a research agenda," in Proc. of 1st Workshop on Everyday Virtual Reality, 2015, pp. 19-22.

[17] X. de Tinguy, C. Pacchierotti, M. Marchal, and A. Lécuyer, "Toward Universal Tangible Objects: Optimizing Haptic Pinching Sensations in 3D Interaction," in Proc. of IEEE Conference on Virtual Reality and 3D User Interfaces, 2019.

[18] M. O. Ernst and M. S. Banks, "Humans integrate visual and haptic information in a statistically optimal fashion," Nature, vol. 415, no. 6870, p. 429, 2002.

[19] L. J. Berryman, J. M. Yau, and S. S. Hsiao, "Representation of object size in the somatosensory system," Journal of Neurophysiology, vol. 96, no. 1 , pp. 27-39, 2006.

[20] F. Argelaguet, L. Hoyet, M. Trico, and A. Lecuyer, "The role of interaction in virtual embodiment: Effects of the virtual hand representation," in Proc. of IEEE International Conference on Virtual Reality, 2016, pp. 3-10.

[21] F. Chinello, C. Pacchierotti, M. Malvezzi, and D. Prattichizzo, "A three revolute-revolute-spherical wearable fingertip cutaneous device for stiffness rendering," IEEE Transactions on Haptics, 2017. 